\title{
Feedback auto-controlado e aprendizagem de uma habilidade motora discreta em idosos
}

\author{
Suzete Chiviacowsky ${ }^{1}$ \\ Franklin L. de Medeiros 2 \\ José F.G. Schild ${ }^{1}$ \\ Mariângela R. A fonso ${ }^{1}$
}

\author{
${ }^{1}$ Escola Superior de Educação Física da \\ U niversidade Federal de Pelotas \\ ${ }^{2}$ Bolsista PIBIC - Universidade Federal de Pelotas
}

\section{RESUMO}

O objetivo do presente estudo foi comparar os efeitos da frequência de conhecimento de resultados (CR) auto-controlada e externamente controlada na aprendizagem de uma habilidade motora discreta em idosos. Vinte e dois idosos na faixa etária de 60 a 76 anos de idade foram utilizados como sujeitos, distribuídos em dois grupos em relação aos diferentes tipos de controle de frequência de CR utilizada: auto-controlada ou externamente controlada. A tarefa consistiu em arremessar um implemento em um alvo. A hipótese testada é que sujeitos que praticam com frequências auto-controladas de CR obtêm maior aprendizagem do que sujeitos que praticam com frequências externamente controladas, conforme resultados anteriores com adultos. A análise dos dados foi realizada através da ANOVA O neway, utilizando-se o pacote estatístico SPSS. Os resultados mostraram forte tendência de superioridade para o grupo com CR auto-controlado, embora a diferença não seja significativa.

Palavras-chave: aprendizagem motora, feedback, frequência autocontrolada, idosos.

\section{ABSTRACT \\ Self-controlled feedback and learning of a discrete motor ability in the elderly}

The objective of the present study was to compare the effects of the selfcontrolled frequency of knowledge of results $(K R)$ and externally controlled frequency in the learning of a discrete motor ability in the elderly. Twenty-two elderly subjects in the age group of 60 to 76 were used as subjects, distributed into two groups in relation to the different types of KR frequency control: self-controlled or externally controlled. The task consisted of throwing an implement at a target. The tested hypothesis is that subjects who practice with self-controlled frequencies of KR obtain higher learning than subjects who practice with externally controlled frequencies, according to previous results with adults. The analysis of the data was carried out through A N OVA Oneway, where the statistical SPSS package was used. The results showed a strong superiority tendency for the group with self-controlled $K R$, although the difference was not significant.

Key-words: motor learning, feedback, self-controlled knowledge of results, elderly 


\section{INTRODUÇÃO}

O conhecimento de resultados (CR), variável de reconhecida relevância para a aprendizagem de habilidades motoras, é uma forma de "feedback" que informa sobre o resultado do movimento em termos do seu objectivo ambiental e possui algumas funções importantes na aprendizagem de habilidades motoras, como a motivacional $(18,24)$, a de orientar o aprendiz em direcção à resposta apropriada (1), assim como a relacional, que possibilita estabelecer relações entre os comandos motores e a resposta que levam ao fortalecimento de esquemas para a produção de novos movimentos (24).

O CR pode ser fornecido de várias formas. Por muito tempo acreditou-se que quanto mais frequentes, mais precisos e mais imediatos, mais eficientes seriam os seus efeitos sobre a aprendizagem de habilidades motoras $(1,5,6,24)$. No entanto esta visão tem sido contrariada por estudos mais recentes. Salmoni, Schmidt, e Walter (21), em um artigo de revisão sobre os efeitos do CR, identificaram alguns experimentos que parecem contrariar esta visão até então predominante mostrando que certas variações do $C R$, que actuam de forma a prejudicar o desempenho durante a fase de aquisição, manifestam um efeito benéfico em testes de retenção e transferência.

Um aspecto importante em relação ao CR é o fato da mai oria dos trabal hos real izados até agora ter utilizado frequências de CR externamente controladas, podendo-se observar praticamente um controle total da situação de aprendizagem por parte do pesquisador, enquanto pouca ou nenhuma ênfase é colocada no aprendiz.

Estudos realizados na área da percepção da auto-eficácia $(2,3)$, da utilização de estratégias de aprendizagem auto-reguladas (8), do auto-estabelecimento de metas (31), assim como da aprendizagem académica (28), vêm indicando que a capacidade de utilizar estratégias cognitivas ou comportamentais, num contexto auto-controlado pelos próprios sujeitos, tem-se mostrado positiva para a aprendizagem. A aprendizagem com auto-controle refere-se a um tipo de situação de aprendizagem onde o aprendiz pode actuar mais activamente no decorrer do processo. Nesta abordagem, o próprio sujeito é quem toma decisões relacionadas às variáveis do processo a serem estudadas, diferente da abordagem anterior, onde pode acontecer a situação de estarmos fornecendo o CR quando o aprendiz não necessita desta informação ou, por outro lado, não a estarmos fornecendo quando ele real mente necessita.

Os primeiros a utilizar esta abordagem, especificamente nos experimentos sobre frequência de CR foram Janelle e seus colaboradores $(17,16)$. Utilizando tarefas discretas em seus estudos (tacada do golfe e arremesso de uma bola ao alvo), os autores compararam grupos que receberam frequências auto-controladas de CR em relação a grupos que praticaram em diferentes condições: com CR sumário a cada cinco tentativas, com $100 \%$ de frequência de CR e com frequência de CR igual ao grupo auto-controlado, mas controlado pelo experimentador. Os resultados foram significativamente superiores para os grupos que receberam um arranjo de CR auto-controlado em relação aos outros grupos. Chiviacowsky e Wulf (9) também confirmaram a superioridade na aprendizagem para sujeitos adultos que receberam um arranjo auto-controlado de CR em relação a um grupo com frequência externamente controlada, numa tarefa sequencial com objectivos espaciais e temporais, de pressionar teclas no teclado numérico do computador.

Entretanto, os efeitos causadores desta vantagem ainda não estão completamente esclarecidos. Wulf e Toole (30) colocam que os grupos com auto-controle devem se engajar em diferentes actividades de processamento que os grupos "yoked" (grupos que recebem CR de forma externamente controlada, equiparados, um a um, aos sujeitos do grupo auto-controlado) as quais foram benéficas para a aprendizagem. Um exemplo, talvez, seria que os sujeitos do grupo auto-controle puderam testar estratégias enquanto escolhiam ou não usar a ajuda física, enquanto os outros podem ter sido desencorajados a fazer isto pela utilização randômica da ajuda física. Chiviacowsky e Wulf $(9,10)$ tentaram compreender quando e porquê os sujeitos solicitavam feedback, quando tinham a oportunidade de fazê-lo, utilizando questionários e análises das tentativas com e sem $C R$. Seus resultados demonstraram que os aprendizes do grupo com auto-controle não solicitam CR de forma aleatória mas, ao contrário, utilizam uma estratégia, que geral mente consiste em utilizar o CR após "boas tentativas" a fim de confirmar que o seu 
desempenho foi (mais ou menos) no alvo. Estes resultados podem demonstrar que arranjos de prática auto-controlados estão mais de acordo com as necessidades ou preferências dos aprendizes do que arranjos externamente controlados, o que pode explicar os benefícios observados na aprendizagem. Parece, assim, que a variável auto-controle apresenta superioridade quando nos referimos à aprendizagem de habilidades motoras em adultos. Entretanto, pouco se sabe sobre os possíveis efeitos desta variável na aprendizagem de habilidades motoras em diferentes níveis de desenvolvimento motor. Em um estudo que verificou os efeitos da variável em questão em crianças (19), os autores utilizaram como sujeitos crianças na faixa etária de 10 anos de idade, encontrando resultados opostos aos estudos com adultos, ou seja, melhor aprendizagem para o grupo que recebeu frequência de CR controlada pelo experimentador. Neste estudo, também foi aplicado um questionário com o objectivo de compreender quando/por que as crianças preferem receber as informações de CR. Os resultados demonstraram concordância com os resultados com adultos (preferência pelo CR após "boas" tentativas), apesar da análise dos erros na fase de aquisição mostrar que as crianças não são tão eficientes quanto os adultos em discriminar entre boas e más tentativas.

Entretanto, nenhum estudo abordando a variável frequência de $C R$ auto-controlada foi ainda realizado em idosos. Sabe-se que, à medida que o indivíduo envelhece, existe um declínio funcional e morfológico do sistema nervoso, levando a modificações nas respostas motoras (14). Assim, o objectivo deste estudo foi verificar os efeitos da frequência auto-controlada de CR nesta faixa de desenvolvimento.

\section{Método}

O bjectivo

Comparar os efeitos da frequência auto-controlada de CR com frequências externamente controladas na aprendizagem de uma habilidade motora discreta em idosos.

\section{A mostra}

A amostra foi constituída de 22 sujeitos, equiparados em relação ao sexo, na faixa etária de 60 a 76 anos de idade, distribuídos em dois grupos de 11 sujeitos, de acordo com os diferentes tipos de fre quência de CR. Todos os sujeitos participaram como voluntários, não possuíam conhecimento sobre o objectivo do experimento e também não possuíam experiência anterior com a tarefa.

Tarefa

A tarefa consistiu em arremessar, por cima do ombro, um saquinho contendo feijão em um alvo fixo colocado no chão. A tarefa foi realizada com o membro não dominante, sem ver o al vo e com os sujeitos na posição sentada de costas para o alvo.

\section{Equipamento e material}

Foi utilizado um alvo circular de $2 \mathrm{~m}$ de diâmetro, impresso em pano e afixado no solo, com o seu centro à uma distância de $2 \mathrm{~m}$ da área de arremesso. $\mathrm{O}$ centro do alvo teve valor 100 e mediu $20 \mathrm{~cm}$; os outros espaços tiveram respectivamente $10 \mathrm{~cm}$ a mais de raio, com valores $90,80,70,60,50,40,30$, 20, 10 e zero. $O$ alvo foi dividido em quatro partes na forma de um $X$, onde foi possível obter a direcção do erro (antes, depois, esquerda, direita). Foram utilizados saquinhos de pano de forma circular, contendo feijão e pesando $100 \mathrm{~g}$. Também foi utilizado um cronómetro digital para controlar os intervalos de tempo (10 s) entre as tentativas.

\section{Delineamento experimental}

Os sujeitos foram distribuídos em 2 grupos desta forma: 11 sujeitos para o grupo que recebeu frequência de CR auto-controlada (grupo AC) e 11 sujeitos para o grupo que recebeu frequência de $C R$ externamente controlada (grupo EC). O grupo EC recebeu frequências de $C R$ equiparadas, sujeito a sujeito com o grupo $\mathrm{AC}$, de forma que o número de CRs solicitados assim como o espaçamento entre as solicitações fosse os mesmos para ambos os grupos. O tempo de espera para apresentação do CR, quando solicitado, foi de $5 \mathrm{~s}$. O tempo pós-CR foi de $5 \mathrm{~s}$. $\mathrm{O}$ intervalo inter-tentativas foi, portanto, de $10 \mathrm{~s}$. A fase de aquisição constou de 60 tentativas de prática. A fase de retenção foi realizada 24 horas após a fase de aquisição e constou de 10 tentativas, sem CR. Nas duas fases estudadas, aquisição e retenção, foram realizadas a análise descritiva dos dados, composta por uma medida de tendência central, repre- 
sentada pela média e uma medida de dispersão. Os dados utilizados para anál ise foram as médias dos erros de cada tentativa. Para a fase de aquisição foram realizadas comparações das médias em blocos de 10 tentativas. Já a fase de retenção constou de apenas um bloco de 10 tentativas.

\section{Procedimentos}

Antes de iniciar a fase de aquisição os sujeitos foram informados que a tarefa teria como objectivo arremessar saquinhos de feijão em um alvo, posicionados de costas para este. Também foi informado que receberiam informações do pesquisador sobre os resultados nas tentativas.

Para os sujeitos que receberam frequência auto-controlada de $\mathrm{CR}$, foi informado que deveriam controlar a sua frequência de $C R$, ou seja, que não receberiam informações de CR a não ser quando solicitassem. Também receberam a instrução para só solicitarem o $\mathrm{CR}$ quando achassem que real mente precisavam do mesmo. Já os sujeitos dos grupos com frequência externamente controlada receberam a informação de que às vezes receberiam a informação de $C R$ e às vezes não, mas que todas as tentativas seriam importantes e seriam utilizadas para posterior análise. Foi explicado também aos sujeitos, que deveriam esperar o aviso do pesquisador para iniciar a realização de cada tentativa.

Todos os dados foram coletados na Escola Superior de Educação Física da Universidade Federal de Pelotas, utilizando salas especialmente reservadas para este fim.

\section{RESULTADOS}

$\mathrm{Na}$ análise dos resultados, as curvas de desempenho foram traçadas em função dos blocos de tentativas, tendo como medida da variável dependente a média dos erros absolutos obtidos em cada bloco. Foram realizadas Análises de Variância (ANOVA) para verificar as eventuais diferenças entre blocos e tipo de $\mathrm{CR}$, nas duas fases do experimento, separadamente para cada fase. Os dados foram analisados através do programa estatístico SPSS.

Fase de aquisição

Pode-se observar (Figura 1, blocos A1 a A6) que o grupo EC melhorou o seu desempenho até o terceiro bloco, piorando no quarto bloco, melhorando novamente do quarto até o sexto bloco. Já o grupo $A C$ demonstrou melhora constante do primeiro ao último bloco. De forma geral, ambos os grupos melhoraram o seu desempenho através das tentativas da fase de aquisição, com tendência de melhor resultado para o grupo EC. Através da ANOVA O neW ay, com medidas repetidas, foram encontradas diferenças significativas entre os blocos $[F(5 ; 100)=4,35$; $p=0,00]$, mas não entre os grupos $[F(1 ; 20)=0,23$; $p=0,63]$ e na interacção entre blocos e grupos $[F(5 ; 100)=1,08 ; p=0,37]$. O teste de Tukey detectou diferenças entre o bloco 1 e o bloco 6 .

Fase de retenção

Para a fase de retenção (Figura 1, bloco R), pode-se constatar diferença no desempenho dos grupos, com forte tendência de menor valor de erro para o grupo AC. A ANOVA O ne-Way entretanto não detectou diferença significativa entre os grupos, $[F(1 ; 20)=3,00 ; p=0,37]$.

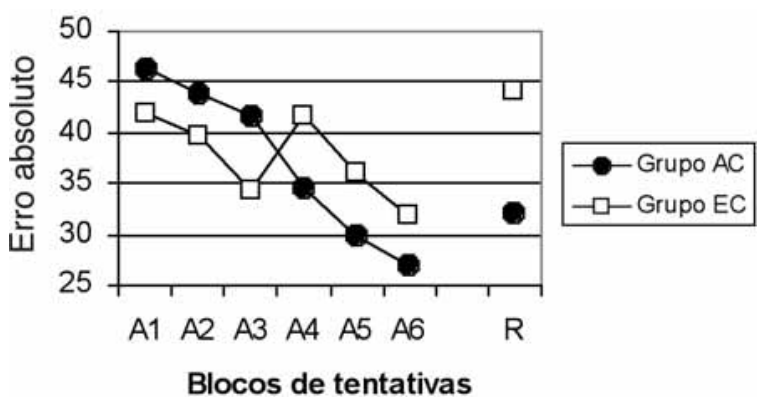

Figura 1. Médias dos grupos, em erro absoluto, nas fases de aquisição e retenção.

\section{DISCUSSÃO E CONCLUSÃO}

Os resultados do presente estudo mostraram-se diferentes dos estudos prévios com adultos $(9,16,17,27)$, já que na variável erro absoluto, apenas tendências e não diferenças significantes de melhores resultados para o grupo $\mathrm{AC}$, em relação ao grupo $\mathrm{EC}$ foram encontradas. Assim, parece que em idosos, ambas as formas de recebimento do CR (auto-controlado e externamente-controlado) desenvolveram a mesma capacidade de desempenho da tarefa aprendida na fase de retenção. 
Tais resultados diferem também de estudos anteriores relacionados à variável frequência de conhecimento de resultados externamente controlada e nível de desenvolvimento motor, já que os estudos com idosos demonstraram resultados similares aos estudos com adultos. Carnahan, Vandervoort e Swanson (7) estudaram a variável sumário de CR (onde todas as informações de CR de um determinado bloco de tentativas é fornecido em conjunto após a execução de todo o bloco) em idosos e encontraram resultados semelhantes aos encontrados em adultos, com CR sumário obtendo melhor aprendizagem do que CR fornecido após cada tentativa. Os autores, no entanto, utilizaram teste de retenção de 10 minutos, onde os efeitos da prática ainda se encontram presentes. Já Behrman, Linden e Cauraugh (4) compararam grupos de idosos que receberam diferentes frequências relativas de CR ( $100 \%$ e $50 \%)$. Os resultados mostraram discreta superioridade da frequência reduzida de $C R$, embora não significativa, o que demonstra que nesta variável os idosos parecem aprender de maneira semel hante aos adultos. Também Wishart e Lee (29) compararam diferentes frequências ( $100 \%$ e $67 \%$ ) e não encontraram diferenças na variável estudada e tampouco interacção entre a frequência de CR e nível de desenvolvimento (adultos e idosos).

De forma similar, estudos recentes têm demonstrado que a aquisição de novas habilidades motoras finas pode ser preservada por dois anos, sem prática durante este intervalo de tempo, de forma similar por adultos de 18 a 95 anos (25). Também Daselaar, Rombouts, Veltman, Raaijmakers e Jonker (11), através de seu estudo mostraram que, apesar de pequenas diferenças terem sido demonstradas na velocidade de aprendizagem entre adultos jovens e idosos, o grau de aprendizagem foi similar para ambos.

Entretanto, alguns estudos sugerem a existência de diferenças entre idosos e adultos em características específicas do comportamento motor, como na capacidade dos idosos de processar informações e de aprender em relação aos adultos (12, 23, 22). Grouios (13) coloca que a velocidade em tempo de reacção é diminuída consideravelmente a partir dos 50 anos de idade. Também em relação ao tempo de movimento, foi comprovado que, com a idade, a mesma acção é realizada em tempos mais prolongados (20). De acor- do com Spirduso (26) a razão principal da redução da velocidade de reacção e de resposta parece ser a considerável diminuição da capacidade de processar informação, de codificar, reconhecer, comparar e selecionar a resposta de acordo com o objectivo e não à degradação das diferentes estruturas cognitivas envolvidas. Ainda, Hedel e Dietz (15), em um estudo que utilizou a aprendizagem de uma tarefa locomotora em adul tos e idosos, demonstraram que os idosos dependem mais das informações visuais do que os adultos. Quando as informações visuais foram propositalmente retiradas, os adultos conseguiram utilizar informações acústicas, tácteis e proprioceptivas para aprendizagem da tarefa, o que não aconteceu com os idosos.

As diferenças encontradas no presente estudo podem demonstrar que os idosos se comportam de forma diferente em relação aos adultos no que se refere aos efeitos das variáveis de aprendizagem motora, entre elas a frequência de conhecimento de resultados auto-controlada. Dessa forma, as vantagens encontradas para o feedback auto-controlado em adultos não podem ser generalizadas para diferentes níveis de desenvolvimento motor. Conclui-se que as frequências auto-controladas podem não proporcionar maior aprendizagem aos idosos em relação a frequências externamente controladas, apesar da forte tendência à superioridade. Sugere-se que mais estudos sejam realizados na área da aprendizagem motora com auto-controle em diferentes níveis de desenvolvimento.

\section{CORRESPONDÊNCIA}

\section{Suzete Chiviacowsky}

Escola Superior de Educação Física

Rua Luís de Camões, 625 - Tablada

CEP 96055-630 Pelotas - RS

suzete@ charqueadasantarita.com.br 


\section{REFERÊNCIAS}

1. Adams, J. A. (1971) A closed-loop theory of motor learning. Journal of M otor Behavior 3: 111-149

2. Bandura, A. (1977). Self-efficacy: Toward a unifying theory of behavioral change. Psychological Review 84: 191-215

3. Bandura, A. (1993). Perceived self-efficacy in cognitive development and functioning. Educational Psychologist 28: 117-148

4. Behrman, A. L., Linden, D. W. V., \& Cauraugh, J. H. (1992). Relative frequency knowledge of results: olders adults learning a force-time modulation task. Journal of $H$ uman M ovement Studies 23: 233-250

5. Bilodeau, E. A., \& Bilodeau, I. M. (1958). Variable frequency of knowledge of results and the learning of a simple skill. Journal of Experimental Psychology 55: 379-383

6. Bilodeau, E. A., Bilodeau, I. M., \& Schumsky, D. A. (1959). Some effects of introducing and withdrawing knowledge of results early and late in practice. Journal of Experimental Psychology 58: 142-144

7. Carnahan, H., Vandervoort, A. A., \& Swanson, L. R. (1996). The influence of summary knowledge of results and aging on motor learning. Research Q uarterly for Exercise and Sport 67(3): 280-287

8. Chen, D., \& Singer, R.N. (1992). Self-regulation and cognitive strategies in sport participation. International Journal of Sport Psychology 23: 277-300

9. Chiviacowsky, S. \& Wulf, G (2002). Self-controlled feedback: Does it enhance learning because performers get feedback when they need it? R esearch Q uartely for Exercise and Sport 73(4): 408-415

10. Chiviacowsky, S. \& Wulf, G (2005). Self-controlled feedback is effective if it is based on the learner's performance. Research Q uartely for Exercise and Sport 76(1): 42-48

11. Daselaar, S.M., Rombouts, S.A.R.B., Veltman, D.J., Raaijmakers, J.G.W. \& e Jonker, C. (2003). Similar network activated by young and old adults during the acquisition of a motor sequence. N eurobiology of A ging 24: 1013-1019

12. Godinho, M., Melo, F., Mendes, R. \& Chiviacowsky, S. (1999). A prendizagem e envelhecimento. Em: Envelhecer melhor com a atividade física. Ed: P. P. Correia, M. Espanha, J. Barreiros. Lisboa: Edições FMH

13. Grouios, G. (1991). Ageing effects on reaction time. International Journal of Physical Education 2: 18-22

14. Haywood, K., Getchell, N. (2004). Desenvolvimento motor ao longo da vida. Porto Alegre: Artmed Editora

15. Hedel, H.J.A, Dietz, V. (2004). The influence of age on learning a locomotor task. Clinical N europhysiology 115: 2134-2143

16. Janelle, C.M., Barba, D.A., Frehlich, S.G., Tennant, L.K., \& Cauraugh, J.H. (1997). Maximizing performance effective- ness through videotape replay and a self-controlled learning environment. R esearch Q uarterly for Exercise and Sport 68: 269-279

17. Janelle, C.M., Kim, J., \& Singer, R.N. (1995). Subject-controlled performance feedback and learning of a closed motor skill. Perceptual and M otor Skills 81: 627-634

18. Magill, R. A. (1989). M otor learning: Concepts and applications ( $3^{a}$ ed.). lowa: Wm. C. Brown

19. Neves, C., Locatelli, L., Oliveira, C., \& Chiviacowsky, S. (2002). A prendizagem motora em crianças: Efeitos da freqüência auto-controlada de conhecimento de resultados. A tas do III Seminário de Comportamento M otor, Gramado, RS

20. Pohl, P. S., Winstein, C.J. \& Fisher, B.E. (1996). The locus of age-related movement slowing: Sensory processing in continuous goal-directed aiming. Journal of Gerontology: Psychological Sciences 51(2): 94-102

21. Salmoni, A., Schmidt, R. A., \& Walter, C. B. (1984). Knowledge of results and motor learning: $A$ review and critical reappraisal. Psychological Bulletin, 95: 355-386

22. Santos, S. \& Tani, G. (1995). Tempo de reação e aprendizagem de uma tarefa de "timing" antecipatório em idosos. Revista Paulista de Educação Física 9: 51-62

23. Santos, S. (1999). Cognitive aspects of movement timing control in old age. Tese de Doutorado. University of Manchester

24. Schmidt, R. A. (1975). A schema theory of discrete motor skill learning. Psychological R eview 82: 225-260

25. Smith, C.D., Walton, A., Loveland, A.D., Umberger, G.H., Kryscio, R.J., \& Gash, D.M. (2005). Memories that last in old age: motor skill learning and memory preservation. Neurobiology of aging 26: 883-890

26. Spirduso, W.W. (1995). Physical dimensions of aging. Champaign, III: Human Kinetics

27. Titzer, R., Shea, J. B., \& Romack, J. (1993). The effect of learner control on the acquisition and retention of a motor task. Journal of Sport \& Exercise Psychology 15 (Supplement): S84

28. Winne, P. H. (1995). Inherent details of self-regulated learning. Educational Psychologist 30: 173-187

29. Wishart, L. R., \& Lee, T.D. (1997). Effects of aging and reduced relative frequency of knowledge of results on learning a motor skill. Perceptual and M otor Skills, 84(3 Pt 1): $1107-22$

30. Wulf, G., \& Toole, T. (1999). Physical assistance devices in complex motor skill learning: Benefits of a self-controlled practice schedule. Research Quarterly for Exercise and Sport 70: $265-272$

31. Zimmerman, B. J., \& Kitsantas, A. (1997). Developmental phases in self-regulation: Shifting from process goals to outcome goals. Journal of Educational Psychology 89: 29-36 